\title{
Automatic and attentional components in perception of size-at-a-distance
}

\author{
WILLIAM EPSTEIN \\ University of Wisconsin-Madison, Madison, Wisconsin \\ and \\ KRISHAN D. BROOTA \\ University of Delhi, Delhi, India
}

\begin{abstract}
It is assumed that a sequence of operations is executed in perceiving size-at-a-distance. The aim of these experiments was to determine the extent to which execution of these operations requires allocation of attention. Three hypotheses were considered: full automaticity-the entire sequence of operations is automatic, proceeding without allocation of attention; partial automaticity-the operations culminating in a representation of projective size and viewing distance are automatic, whereas the combinatorial operation culminating in a distally correlated representation of size require attention; and zero automaticity -all of the operations require attention. To decide among these hypotheses, we had subjects perform forced-choice size recognition tests under two conditions. In the size-directed condition, the subjects were motivated to process size. In the numerosity-directed condition, subjects were motivated to direct attention to discrimination of numerosity, thereby causing attention to be diverted from processing of size. Examination of the pattern of choices on the recognition test showed results that conformed best to the hypothesis of partial automaticity.
\end{abstract}

One long-held view about the perception of shape-ata-slant and size-at-a-distance is that these perceptual descriptions are governed by a sequential set of operations. (Various expositions of this view can be found in Baird, 1982; Baird \& Wagner, 1982; Epstein, 1973; Epstein \& Hatfield, 1978; Epstein, Hatfield, \& Muise, 1977; Gogel, 1978; Goldstein, 1984. ch. 9; Rock, 1977, 1983; Wallach \& Frey, 1972; among others). In the version explicated by Epstein et al., the initial operations involve registration of the projective shape or size and discrimination of the optical correlates of slant-in-depth or viewing distance. The products of these operations are representations of projective shape and slant-in-depth and representations of projective size and viewing distance, respectively. ${ }^{1}$ Subsequent operations are presumed to compute a distally correlated shape and size by combining the information in these representations according to certain rules, which are generally referred to as the shapeslant invariance algorithm and the size-distance invariance algorithm.

In a recent study, Epstein and Lovitts (1985) sought to assess the attentional demands of the various operations presumed to underlie perception of shape-at-a-slant. Their experiments were designed to distinguish among three

K. D. Broota's work on this project was supported by a Fulbright Senior Fellowship and a grant to William Epstein from the Graduate School of the University of Wisconsin. Requests for reprints should be addressed to William Epstein at the Department of Psychology, University of Wisconsin, Madison, Wisconsin 53706. hypotheses: full automaticity-all of the operations are automatic; they are mandatory and make no demands on attentional resources; partial automaticity-the initial operations are automatic but the later, combinatorial operation is not mandatory and requires allocation of attentional resources; zero automaticity-none of the operations are automatic; all require allocation of attention.

Epstein and Lovitts (1985) compared subjects' performance on a forced-choice task under two attentional conditions, one designed to focus attention on processing shape-at-a-slant, the other designed to withdraw attention from processing shape-at-a-slant. The fundamental comparison involved the subjects' choices between an objective and a projective match for a previously viewed shape that had been rotated in depth. Epstein and Lovitts reasoned that if full automaticity correctly characterized the process, then under both attentional conditions, there should be a significant preference for the objective match; if zero automaticity was the correct characterization, the shape-directed attentional conditions should yield a significant preference for objective matches, whereas the contrasting attentional condition should yield an even division between objective and projective choices; finally, if partial automaticity was the correct characterization, objective matches should be preferred under the shapedirected attentional condition, but the preference should be reversed when attention was withdrawn; that is, projective choices should be preferred over objective choices. The latter prediction follows from the claim that the automatic operations yield a representation of projective 
shape and that computation of a distally correlated shape, which would lead to a preference for the objective match, is not carried out when attention is withdrawn. The results of Epstein and Lovitt's experiments corroborated the partial-automaticity hypothesis.

In the present report we describe two experiments that exploited the same logic to assess automatic and attentional components in the perception of size when viewing distance varies. We will show that when forced to choose between an objective size match and a projective size match for a previously viewed standard, subjects who have been directed to attend to size choose objective size matches, whereas subjects exposed to the same standard stimuli under conditions that withdraw attention from processing size prefer projective size matches.

\section{EXPERIMENT 1}

\section{Method}

\section{Subjects}

The volunteer subjects were 60 university students. Thirty were randomly assigned to the size-directed attentional condition, and the other 30 to the numerosity-directed attentional condition. The ages of the students ranged from 20 to 24 years. All had normal or corrected-to-normal vision.

\section{Stimuli}

The standard stimuli were eight white posterboard squares, sized $6,7,8,9,10,11,12$, and $13 \mathrm{~cm}$. Each standard was individually mounted on a thin stalk. The center of each square was at a constant height, aligned with the subject's eye level. Starting at a point $3 \mathrm{~mm}$ from the midpoint of the base of each square, a vertical array of black dots, each $3 \mathrm{~mm}$ in diameter, was drawn along the vertical axis of each square at 3-mm intervals. The number of dots in the vertical array varied between 3 and 7 . The number of dots assigned to each square was randomly determined. Each of the eight squares was presented at three viewing distances: $1,1.5$, and $2 \mathrm{~m}$. The visual angles subtended by the squares varied from $.7^{\circ}$ to $.16^{\circ}$. The 24 size-distance combinations ( 8 sizes $\times 3$ distances) were randomly divided into six sets of 4 , taking care that within each set no size was repeated.

The test figures were pairs of different-sized squares drawn side by side, each pair presented on an individual test sheet. The squares presented on the test were of four types: objective size matches $(O)$, projected size matches $(P)$, narrow foils $\left(F_{n}\right)$, and wide foils $\left(F_{w}\right)$. The objective-size test square exactly matched the size of one of the four squares presented during the viewing trials; the projectivesize test alternative was a square that matched the projective size of the standard square at the viewing window; the narrow foil was a square $1 \mathrm{~cm}$ narrower than the projective size; and the wide foil was a square $1 \mathrm{~cm}$ wider than the objective size. Thus, there were five types of test pairs: $\mathrm{O}-\mathrm{P}, \mathrm{O}-\mathrm{F}_{\mathrm{n}}, \mathrm{O}-\mathrm{F}_{\mathrm{w}}, \mathrm{P}-\mathrm{F}_{\mathrm{n}}$, and $\mathrm{P}-\mathrm{F}_{\mathrm{w}}$. A complete test consisted of four presentations of each of the five test pairs; for two trials in each set of four the left-right spatial arrangement was as shown above, and for the other trials the left-right arrangement was reversed. The presence of foils ensured that neither the objective match nor the projective match occupied a fixed position in the test set. The objective match was widest and intermediate in size equally often, and the projective match was narrowest and intermediate equally often.

The test sheets were viewed at the subject's self-determined reading distance. Inasmuch as reading distances vary among subjects and, very likely, for a given subject between trials, the projective sizes of the test squares were variable. This fact may seem troublesome in considering the projective-size test alternative. However, the logic of our measurement procedure does not require that the projective size of the test squares be fixed, or that the projective size of the test square that is offered as a projective option actually match the projective size of the standard. What is required by our measurement procedure is that the perceived size of the projectivetest alternative $\left(S_{t}^{\prime}\right)$ match the perceived size of the standard $\left(S_{S}^{\prime}\right)$ when $S_{S}^{\prime}$ is determined by the projective size of the standard. In order to satisfy this requirement, we computed the projective size of the standard at the viewing window and presented as the projective option on the two-alternative forced-choice test a square that would yield the same $S^{\prime}$. In determining the physical size of this projective test option, we assumed that under the full-cue, unrestricted-viewing-time concitions, $S^{\prime}$ for the test squares would be veridical, and we chose the dimensions of the projective-size test option to match the projective dimensions of the standard.

\section{Apparatus}

The main components of the apparatus were a display unit and a device to control the exposure duration of the standard square. The display unit was an extended unpainted wooden board with a rich wood texture, $360 \mathrm{~cm}$ long and $90 \mathrm{~cm}$ wide. An upright black wooden panel at the far end served as a backdrop for the display. Another upright panel at the near end was equipped with an extended padded viewer and shutter. Normal fluorescent room illumination prevailed. A camera-type exposure device was fixed for each of the two eyes on the other side of the viewing window, which allowed an exposure of the targets for a duration of $1 \mathrm{sec}$ with the pressing of an extended shutter cord.

\section{Procedure}

Ten subjects were randomly assigned to each of the six sets of size-distance combinations. Each set contained four squares of different sizes; two of the squares were assigned to one of the three viewing distances, and the remaining two squares were assigned to the remaining two viewing distances. Five subjects in each of the six groups were assigned randomly to the size-directed attentional condition, and five subjects were assigned to the numerosity-directed attentional conditions. In both attentional conditions the subjects viewed the standard and test squares binocularly under full-cue conditions.

Size-directed condition. Each subject assigned to the size-directed condition was told that four squares of different sizes would be presented briefly, one at a time, at different distances. The task assigned to the subject was to discriminate among the squares in preparation for a subsequent test that would require recognition of previously presented sizes. The subject was instructed to ignore the dots; the presence of the dots was attributed to the requirements of a different experiment. Administration of the instructions was followed by five viewing trials. A trial consisted of presentation of the four squares in the set at their assigned distances. The sizedistance combinations remained unchanged over the five trials, but the order of presentation of the size-distance combinations was randomized from trial to trial. The exposure duration for each standard was $1 \mathrm{sec}$; the interval between presentations averaged about $5 \mathrm{sec}$. At the conclusion of these viewing trials, the test booklet, containing 20 sheets, was given to the subject. The subject was informed that each sheet in the booklet contained a pair of squares of different sizes and that each test pair included one of the four squares observed during the viewing trials. The subject was asked "to determine which of the two sizes comes closest to matching one of the four sizes you observed during the viewing trials." The subject indicated a choice by marking one of the squares in the pair. After making a choice, the subject was instructed to move on to the next sheet in the booklet. Testing was self-paced, although the subject was encouraged to work quickly.

Numerosity-directed condition. The subjects assigned to the numerosity-directed attentional condition were told that the ex- 
perimental task was an odd-even discrimination. The nature of the task was explained with the help of a rectangular form that was not a member of the stimulus set. The subjects were instructed to respond "odd" or "even" verbally, as quickly as possible upon presentation of the dots. After the instructions were given, the square targets were presented. A numerosity discrimination was secured for each presentation of the standard square. Each subject in the numerosity-directed condition was yoked to a subject in the sizedirected condition so that the pairs of yoked subjects were exposed to the same standards in the same order. At the conclusion of the fifth viewing trial, the size-discrimination test was introduced. The information about the test and the instructions were the same as those provided to the size-directed subjects.

\section{Results}

The critical data for evaluating the contrasting hypotheses are provided by comparing the distribution of choices on the O-P trials under the two attentional conditions. Under the circumstances of the experiment it seems unlikely that the choices on the O-P trials reflect perfectly the perceived sizes of the standards during the viewing trials. Factors extraneous to the process of perceiving size at a distance, such as memorial factors, probably contribute to determining performance. These factors cannot be ruled out; however, the observed frequencies of choices of $\mathrm{O}$ and $\mathrm{P}$ on the O-P trials may be adjusted by taking into account these extraperceptual factors.

The subjects' choices on the O-F and P-F trials were used for this purpose, the former to adjust the obtained $O$ frequencies under both attentional conditions and the latter to adjust the obtained $P$ frequencies under both attentional conditions. The reasoning underlying the adjustment procedure was that the O-F and P-F test trials, which offered the subject a choice between an appropriate match $(\mathrm{O}$ or $\mathrm{P}$ ) and a size (F) never encountered during the viewing trials, provide a basis for estimating the influence of extraneous factors on O-P test performance. Performance on the O-F trials is taken as a measure of the greatest preference for $\mathrm{O}$ that can be expected on the O-P trials, taking all factors into account, given that the perceived size during the viewing trials corresponded to the objective size. Analogously, performance on the P-F trials is taken as a measure of the greatest preference for $P$ on the O-P trials, taking all factors into account, given that the perceived size during the viewing trials corresponded to projective size. In the absence of other factors, a subject who perceived the objective sizes of the standards should choose $\mathrm{O}$ on all $\mathrm{O}-\mathrm{F}$ trials and a subject who perceived the projective sizes of the standards should choose $P$ on all P-F trials. The performances of these hypothetical subjects on the O-P trials can be accepted as accurate reflections of perceived size and require no adjustment. However, an adjustment is required for subjects whose performance on the O-F or P-F trials or both deviates from perfect preference. The number of $O$ choices on the $O-P$ trials for each individual subject under the two attentional conditions was adjusted by subtracting the value yielded by calculating $(1-\mathrm{O} / \mathrm{O}+\mathrm{F})$ based on the $\mathrm{O}-\mathrm{F}$ trials. The number of $P$ choices for each individual subject on the $O-P$ trials under the two attentional conditions was adjusted by subtracting the value yielded by calculating $(1-P / P+F)$ based on the P-F trials. The mean adjusted scores are shown in Figure 1.

The trends exhibited in Figure 1 are clear. The first analyses assessed the difference between the adjusted $O$ scores and between the adjusted $P$ scores separately under the two attentional conditions. The adjusted $\mathrm{O}$ score was higher under the size-directed condition (mean = 2.86) than under the numerosity-directed condition (mean $=1.23$ ); the adjusted $P$ score was higher under the numerosity-directed condition (mean $=2.16$ ) than under the size-directed condition (mean $=0.54$ ). Two analyses of variance established that these two differences were significant [for $\mathrm{O}$ scores, $F(1,58)=37.77, p<.001$; for P scores, $F(1,58)=32.5, p<.001]$.

Examination of the individual subjects' adjusted scores showed that in the size-directed condition $80 \%$ of the subjects selected more Os than Ps, $13 \%$ selected more Ps than Os, and 7\% chose an even number of Os and Ps. In the numerosity-directed condition, $60 \%$ of the subjects selected more Ps than Os, $33 \%$ chose more Os than Ps, and $7 \%$ chose an even number of Os and Ps. Thus, the individual subjects' responses corroborate the outcome of the analysis of variance.

The second analysis compared the adjusted $\mathrm{O}$ and $\mathrm{P}$ scores within the attentional conditions. Under the sizedirected condition the adjusted $O$ scores were significantly higher than the P scores $[F(1,29)=37.26, p<.001]$. On the other hand, in the numerosity-directed condition the adjusted $\mathrm{P}$ scores were higher than the $\mathrm{O}$ scores $[F(1,29)=5.87, p<.05]$.
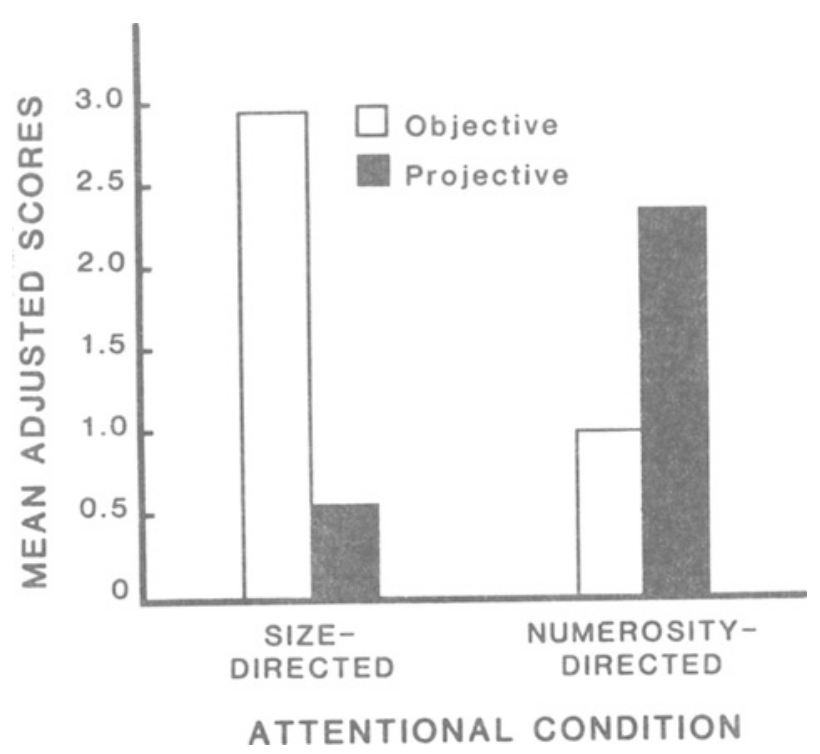

Figure 1. Mean adjusted $O$ scores and mean adjusted $P$ scores on the O-P test trials under the two attentional conditions in Experiment 1 . 
The average percentage of correct answers for the numerosity-discrimination task under the numerositydirected condition was $89 \%$. This indicates that the subjects were allocating processing resources to the assigned task (odd-even discrimination).

\section{Conclusions}

The results of Experiment 1 show that objective matches were preferred under the size-directed condition, whereas projective matches were preferred under the numerosity-directed condition. The outcome is compatible with the hypothesis of partial automaticity proposed by Epstein and Lovitts (1985) in their investigation of the effect of attentional components in the study of shape-ata-slant. According to this hypothesis, only under the sizedirected condition will the subject complete the entire sequence of operations culminating in a distally correlated representation of size, thus leading to a preference for objective size matches. In the numerosity-directed condition, when attention has been withdrawn from processing of size, the subject completes only the early (automatic) operations culminating in proximally correlated representation of size, thus leading to a preference for projective size matches. This hypothesis leads to the expectation that the difference between the preferences in the two conditions should be further accentuated when the numerositydirected condition is made more demanding. Accentuation is expected because an increase in the demands of numerosity discrimination should lead to more complete withdrawal of attention from size-processing, resulting in greater preference for projective size matches.

\section{EXPERIMENT 2}

In Experiment 2, we made the attentional task more demanding by introducing uncertainty as to the orientation and location of the dots on the surface of the target in the numerosity-directed condition.

\section{Subjects \\ Method \\ The volunteer subjects were 60 university students. Thirty were randomly assigned to the size-directed condition and the other 30 to the numerosity-directed condition. None of these subjects had previous experience with the experimental procedure.}

\section{Stimuli}

The standard stimuli were the same eight squares used in Experiment 1. On each of the eight squares was drawn an array of black dots that varied in number, orientation, and location. The number of dots varied randomly from 3 to 7 , as in Experiment 1. The two orientations were vertical and horizontal. The four locations (the starting points of the arrays of dots) were the four midpoints on the perimeter of the square. That is, the vertical arrays of dots were aligned either with the base or with the upper side of the square; the horizontal arrays of dots were aligned either with the left side or with the right side of the square. The position and orientation of the array of dots were also randomly determined for each square. The other aspects of the stimuli were the same as in Experiment 1.

\section{Procedure}

The procedure followed the procedure of Experiment 1 in all respects. The subjects in the size-directed condition were directed to process size and ignore the dots. The subjects in the numerositydirected condition were directed to discriminate odd from even arrays of dots; no mention was made of size perception.

\section{Results}

The pattern of adjusted scores shown in Figure 2 is the same as the pattern obtained in Experiment 1. The adjusted $O$ score was higher under the size-directed condition (mean $=3.23$ ) than under the numerosity-directed condition (mean $=0.33$ ), whereas the adjusted P score was higher under the numerosity-directed condition (mean $=3.03$ ) than under the size-directed condition (mean $=$ 0.34 ). Two analyses of variance established that these two differences were significant [for $\mathrm{O}$ scores, $F(1,58)=$ $210.65, p<.001$; for $P$ scores, $F(1,58)=152.83, p<$ $.001]$.

Examination of the individual subjects' adjusted scores showed that in the size-directed condition $97 \%$ of the subjects selected more Os than Ps and 3\% selected an even number of Os and Ps; no subject selected more Ps than Os. In the numerosity-directed condition, $90 \%$ of the subjects selected more Ps than Os, $7 \%$ selected more Os than Ps, and 3\% chose an even number of Os and Ps. Thus, the individual subjects' responses corroborate the outcome of the analysis of variance.

A comparison of the $O$ and $P$ scores within each attentional condition for the size-directed condition showed that

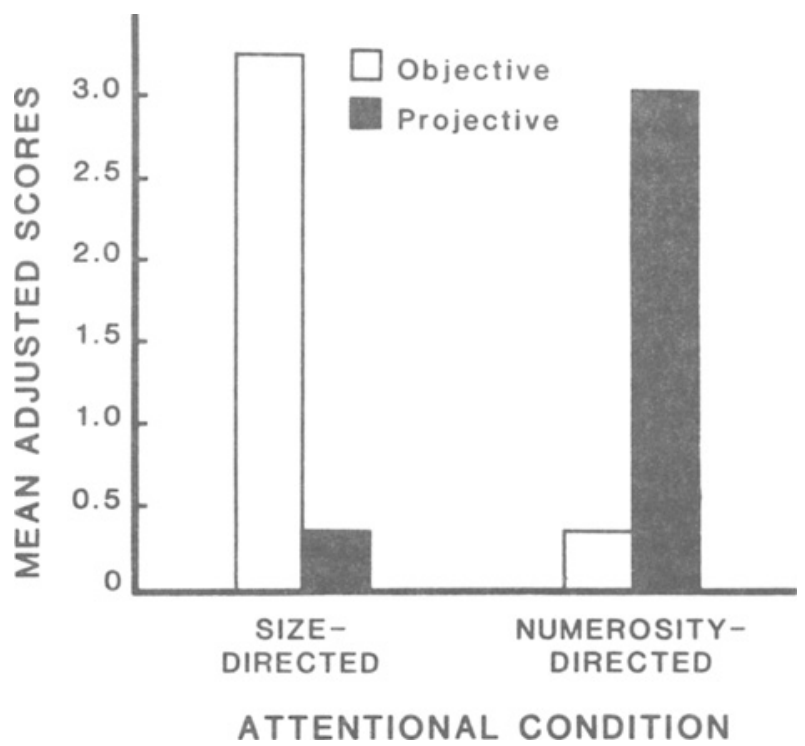

Figure 2. Mean adjusted $O$ scores and mean adjusted $P$ scores on the O-P test trials under the two attentional conditions in Experiment 2. 
the adjusted $\mathrm{O}$ scores were significantly higher than the $P$ scores $[F(1,29)=102.09, p<.001]$; for the numerosity-directed condition, the preference was reversed, and the adjusted $P$ scores were higher than the $\mathrm{O}$ scores $[F(1,29)=81.54, p<.001]$.

The average percentage of correct answers for the numerosity discrimination was $91 \%$. This indicates that the subjects were allocating processing resources to the assigned task.

\section{Conclusions}

The results of Experiment 2 indicate that an increase in demands on the attentional resources accentuates the effect observed in Experiment 1. Figure 3 allows ready comparison of the results of the two experiments. The important observation is that the adjusted $P$ score (mean = 3.03) in the numerosity-directed condition was higher in Experiment 2 than in Experiment 1 (mean $=2.16$ ) $[F(1,58)=11.5, p<.01]$, whereas the adjusted $O$ scores in the size-directed conditions in the two experiments did not differ significantly.

\section{DISCUSSION}

Three characterizations of processing size-at-a-distance were set out in the introduction. The results of the present experiments are best accommodated by the partial-automaticity hypothesis. According to this hypothesis, the operations that culminate in a distally correlated representation of size require allocation of attention. When attention is withdrawn, only the results of the earlier, automatic, operations are available. The products of the automatic operations are assumed to be a representation of projective size and a representation of viewing distance. It follows that allocation and withdrawal of attention should affect reports of perceived size in a systematic way. When attention is directed to size processing, reports of perceived size should be distally correlated; that is, objective size matches should be preferred. But when attention is withdrawn from size processing, reports of perceived size should be proximally correlated; that is, projective size matches should be preferred. The results of both experiments conformed to this pattern. Furthermore, a manipulation designed to increase the demands of the distractor task, and thus more adequately ensure withdrawal of attention from size-processing, did lead to stronger preferences in the direction consistent with the partial-automaticity hypothesis.

The conclusions offered above are consistent with the conclusions offered by Epstein and Lovitts (1985) in their study of automatic and attentional components in perceiving shape-at-a-slant. Inasmuch as the information, representations, and operations that are presumed to underlie perception of size-at-a-distance and shape-at-a-slant have much in common, the agreement between the implications of the earlier and present studies is not surprising. In general, we would expect to observe related effects of variations in allocation of attention whenever it is plausible to infer that a process similar to the one involved in perceived shape-at-a-slant and size-at-a-distance is engaged.

As an example, consider stereoscopic depth. One account (Cormack, 1984; Epstein, 1973; H. Ono \& Comerford, 1977, pp. 112-116; Wallach, Gilliam, \& Cardillo, 1979; Wallach \& Zuckerman, 1963) of perceived stereoscopic depth proposes that disparity and perceived distance are combined to compute depth in a manner

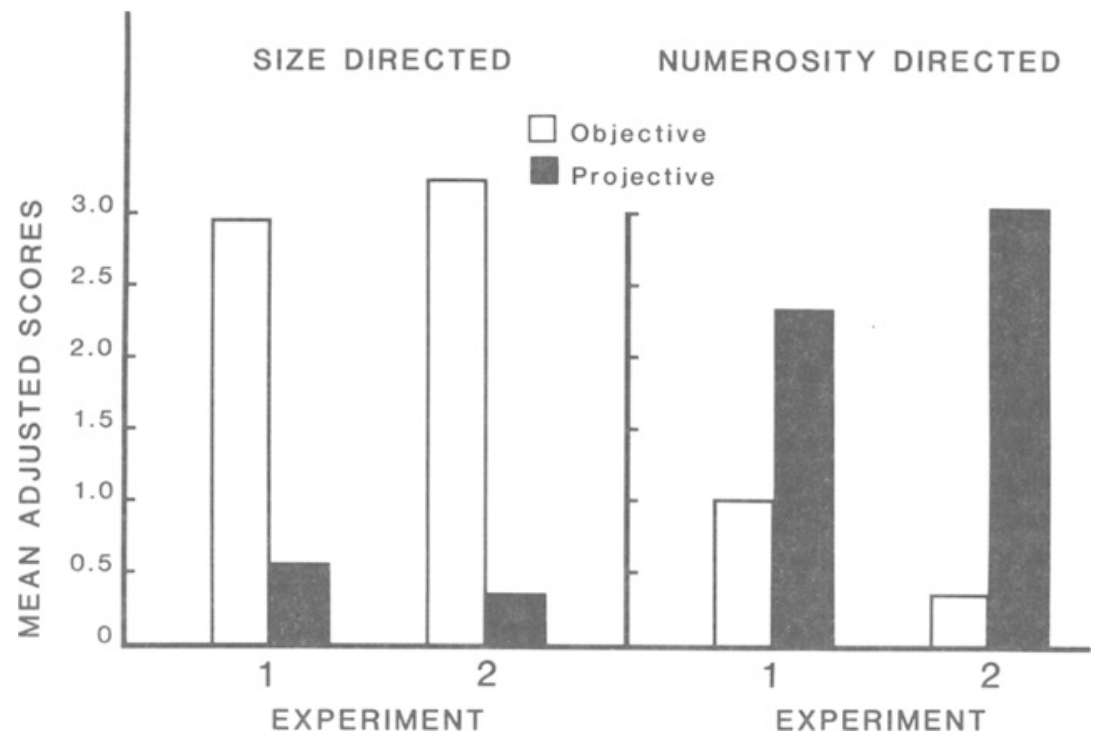

Figure 3. Comparison of results for Experiments 1 and 2. 
analogous to the processing of size-at-a-distance. This account is motivated by the demonstration that the disparity associated with a depth interval is inversely related to the square of the viewing distance ${ }^{2}$ and by the experimental finding of stereoscopic depth constancy. Our elaboration of this account would assign attention a role in determining perceived stereoscopic depth and stereoscopic depth constancy. Consistent with this assignment, which we have made in our work, we would assume that allocation of attention to the resolution of depth is necessary for the computation of distally correlated depth intervals. One test of our view could be carried out in the context of an experimental assessment of stereoscopic depth constancy. If attention is withdrawn from processing depth, we would expect a breakdown of constancy.

A strictly analogous analysis can be carried out for the relationship between motion parallax and perceived depth. The effectiveness of motion parallax has long been recognized, and there have been a number of very compelling contemporary demonstrations of the potency of motion parallax (in particular, see Rogers \& Graham, 1979). For the same reasons that apply in the cases of perceived size and stereoscopic depth, it seems necessary to suppose that for motion parallax to specify internal depth the visual system must take into account absolute distance information. In a recent investigation, M. E. Ono, Rivest, and H. Ono (1986) reported the results of three experiments that demonstrate that the visual system does in fact calibrate motion parallax according to perceived absolute distance in processing depth. As in the cases of perceived shape, size, and stereoscopic depth, we suspect that a place will have to be made for attention in the model of computing motion parallax depth.

There is an alternative to the partial-automaticity account of our current findings and our earlier results for perception of shape-at-a-slant. A number of investigators (Coren \& Porac, 1983; Gogel, 1967; Gogel, Loomis, Newman, \& Sharkey, 1985; Gogel \& Tietz, 1976; Peterson, 1986; Tsal, 1984) have shown that attention allocation can modify the relative effectiveness of different sources of visual information, for example, distance cues in a multiple-cue setting. As an extension of this finding, suppose that withdrawal of attention from processing size-at-a-distance is accompanied by abstention from processing information for distance. Under such circumstances, the likely consequence is that the standard targets will appear to be at the distance of the viewing aperture. (See Gogel, 1977, on the "equidistance tendency.") If this is the effect of withdrawal of attention, then the projective-size preference observed under the numerosity-directed condition would follow from the standard size-distance invariance algorithm (Epstein, 1973). Note that in this interpretation, the same complete process generates the size preferences under both the shapedirected and numerosity-directed conditions. The different size preferences under the two conditions are due to the effect of attention allocation on perceived distance. Inasmuch as our model supposes that distance information is processsed, measures of perceived distance under the two attentional conditions should provide a basis for deciding between these alternatives.

\section{REFERENCES}

BaIRD, J. C. (1982). The moon illusion: II. A reference theory. Journal of Experimental Psychology: General, 111, 304-315.

BaIRD, J. C., Wagner, M. (1982). The moon illusion: I. How high is the sky? Journal of Experimental Psychology: General, 111, 296-303.

Coren, S., \& Porac, C. (1983). The creation and reversal of the Mueller-Lyer illusion through attentional manipulation. Perception, 12, 49-54.

CoRmack, R. H. (1984). Stereoscopic depth perception at far viewing distances. Perception \& Psychophysics, 35, 423-428.

EPSTEIN, W. (1973). The process of "taking-into-account" in visual perception. Perception, 2, 267-285.

EPSTEIN, W., \& HATFIELD, G. (1978). Functional equivalence of masking and cue reduction in perception of shape at a slant. Perception \& Psychophysics, 23, 137-144.

Epstein, W., Hatfield, G., \& Muise, G. (1977). Perceived shape at a slant as a function of processing time and processing load. Journal of Experimental Psychology: Human Perception \& Performance, 3, 473-483.

EPSTEIN, W., \& LovirTs, B. E. (1985). Automatic and attentional components in perception of shape-at-a-slant. Journal of Experimental Psychology: Human Perception \& Performance, 11, 355-366.

Gogel, W. C. (1967). Cue enhancement as a function of task set. Perception \& Psychophysics, 2, 455-458.

GoGEL, W. C. (1977). The metric of visual space. In W. Epstein (Ed.), Stability and constancy in visual perception: Mechanisms and processes (pp. 129-182). New York: Wiley.

GoGEL, W. C. (1978). Size, distance, and depth perception. In E. C. Carterette \& M. P. Friedman (Eds.), Handbook of perception: Vol. 9. Perceptual processing (pp. 299-333). New York: Academic Press. Gogel, W. C., Loomis, J. M., Newman, N. J., \&harkey, T. J. (1985). Agreement between indirect measures of perceived distance. Perception \& Psychophysics, 37, 17-27.

GOGEL, W. C., \& TIETZ, J. D. (1976). Adjacency and attention as determiners of perceived motion. Vision Research, 16, 839-845.

GoLDSTEIN, E. B. (1984). Sensation and perception (2nd ed.). Belmont, CA: Wadsworth.

Ono, H., \& ComerFord, J. (1977). Stereoscopic depth constancy. In W. Epstein (Ed.), Stability and constancy in visual perception: Mechanisms and processes (pp. 91-128). New York: Wiley.

ONo, M. E., Rivest, J., \&No, H. (1986). Depth perception as a function of motion parallax and absolute-distance information. Journal of Experimental Psychology: Human Perception \& Performance, 12, 331-337.

Peterson, M. A. (1986). Illusory concomitant motion in ambiguous stereograms: Evidence for nonstimulus contributions to perceptual organization. Journal of Experimental Psychology: Human Perception \& Performance, 12, 50-60.

Rock, I. (1977). In defense of unconscious inference. In W. Epstein (Ed.), Stability and constancy in visual perception (pp. 321-374). New York: Wiley.

Rock, I. (1983). The logic of perception. Boston: Bradford Press.

Rogers, B., \& Graham, M. (1979). Motion parallax as an independent cue for depth perception. Perception, 8, 125-134.

TsaL, Y. (1984). A Mueller-Lyer illusion induced by selective attention. Quarterly Journal of Experimental Psychology, 36A, 319-333.

WALLACH, H., \&REY, R. (1972). Adaptation to distance based on oculomotor cues. Perception \& Psychophysics, 11, 77-83.

Wallach, H., Gillam, B., \& Cardillo, L. (1979). Some consequences of stereoscopic depth constancy. Perception \& Psychophysics, 26, 235-240.

WALLACH, H., \& ZUCKERMAN, C. (1963). The constancy of stereoscopic depth constancy. The American Journal of Psychology, 76, 404-412. 


\section{NOTES}

1. What is supposed is that in the early stage of processing, operations on optical input detect the properties of the retinal image and generate an abstract representation of these properties. This representation need not be analogic, that is, having the nature of a picture, it need only be one that can be mapped onto an imagistic response system. The notion seems to most closely approximate Rock's ideas (1983, pp. 254269) on the dual aspects of perception.
2. The inverse square law is $\delta=d i / D^{2}$, where $\delta$ is the binocular disparity (in radians), $d$ is the depth interval, $D$ is the viewing distance, and $i$ is the interocular separation.

(Manuscript received March 5, 1986; revision accepted for publication August 5, 1986.) 